\title{
Dynamic bifurcations: hysteresis, scaling laws and feedback control
}

\author{
N. Berglund \\ School of Physics, Georgia Institute of Technology \\ Atlanta GA 30332-0430, USA.
}

December 1, 1999

\begin{abstract}
We review some properties of dynamical systems with slowly varying parameters, when a parameter is moved through a bifurcation point of the static system. Bifurcations with a single zero eigenvalue may create hysteresis cycles, whose area scales in a nontrivial way with the adiabatic parameter. Hopf bifurcations lead to the delayed appearance of oscillations. Feedback control theory motivates the study of a bifurcation with double zero eigenvalue, in which this delay is suppressed.
\end{abstract}

\section{Introduction}

Many physical systems are described by ordinary differential equations (ODEs) of the form

$$
\frac{\mathrm{d} x}{\mathrm{~d} t}=F(x, \lambda),
$$

where $x \in \mathbb{R}^{n}$ is a vector of dynamic variables and $\lambda \in \mathbb{R}^{p}$ a set of parameters. When modeling the system by the equation (11) we implicitly assume that the parameters are kept constant, while only the dynamic variables change in time. There are, however, situations in which the variables considered as parameters change slowly in time, for instance:

- parameters which may be difficult to control, such as the temperature in a chemical reactor or the climate influencing an ecological system;

- control parameters, such as the temperature difference in a convection experiment or the supply voltage of an electrical device, which are slowly varied in order to determine the bifurcation diagram experimentally;

- slowly time-dependent forcings such as a periodic magnetic field acting on a magnet. It is thus important to understand the relation between solutions of the $p$-parameter family of autonomous ODEs (11), and the time-dependent system

$$
\frac{\mathrm{d} x}{\mathrm{~d} t}=F(x, G(\varepsilon t))
$$

where the function $G(\tau): \mathbb{R} \rightarrow \mathbb{R}^{p}$ is given, $\varepsilon$ is a small parameter, and $\tau=\varepsilon t$ is called slow time. It is convenient to rewrite (2) as the singularly perturbed system

$$
\varepsilon \frac{\mathrm{d} x}{\mathrm{~d} \tau}=F(x, G(\tau))=f(x, \tau) .
$$


There is a large literature on equations of this type, see for instance the textbooks [1], 2], the proceedings [3] and the paper [4] for reviews of different approaches.

We will focus here on relations between the solutions of the slowly time-dependent system (3) and the bifurcation diagram of the "frozen" system (11). It turns out that the dynamics near nonbifurcating equilibrium branches is relatively simple and well understood. We summarize some of the relevant results in Section 2. Bifurcations, however, lead to new interesting phenomena, some of which we will review in the next sections.

- Bifurcations in which a single eigenvalue becomes equal to zero may lead to relaxation oscillations [5], bifurcation delay and hysteresis [6, 7]. In Section 3, we present some results on the $\varepsilon$-dependence of the hysteresis area.

- The Hopf bifurcation, in which a pair of complex conjugate eigenvalues crosses the imaginary axis, leads to the delayed appearance of large amplitude oscillations. In Section 4 we present some of Neishtadt's results [8, 9] on the determination of the bifurcation delay.

- Finally, in Section 5, we give a result on a bifurcation problem with a double zero eigenvalue, which arises in the context of feedback control [10, 11.

\section{Non-bifurcating equilibria}

If we naively replace $\varepsilon$ by 0 in equation (3), we obtain the algebraic equation $f(x, \tau)=0$ which defines the equilibrium branches $x=x^{\star}(\tau)$ of the frozen system. One can thus expect that for small positive $\varepsilon$, some solutions of the slowly time-dependent system stay close to some equilibrium branch $x^{\star}(\tau)$. This can be made precise in the following way.

We consider the equation

$$
\varepsilon \frac{\mathrm{d} x}{\mathrm{~d} \tau}=f(x, \tau)
$$

under the following assumption.

Assumption 1. There exist an interval $I \in \mathbb{R}$, which need not be bounded, a neighbourhood $\mathcal{D}$ of the origin in $\mathbb{R}^{n}$, and positive constants $a_{0}, w_{0}, d$ and $M$ such that the following properties are satisfied uniformly in $\tau \in I$.

- The function $f(x, \tau): \mathcal{D} \times I \rightarrow \mathbb{R}^{n}$ is of class $\mathcal{C}^{k}, k \geqslant 2$.

- There exists a function $x^{\star}(\tau): I \rightarrow \mathcal{D}$ such that $f\left(x^{\star}(\tau), \tau\right)=0$, with its derivative $\mathrm{d}_{\tau} x^{\star}(\tau)$ bounded in norm by $w_{0}$.

- The matrix $A(\tau)=\partial_{x} f\left(x^{\star}(\tau), \tau\right)$ has eigenvalues $a_{j}(\tau), j=1, \ldots, n$, such that $\left|\operatorname{Re} a_{j}(\tau)\right| \geqslant a_{0}>0$ for all $j$ (hyperbolicity).

- The function $b(y, \tau)=f\left(x^{\star}(\tau)+y, \tau\right)-A(\tau) y$ is bounded by $M\|y\|^{2}$ for $\|y\| \leqslant d$.

Theorem 1. Under Assumption 1, there exist strictly positive constants $\varepsilon_{0}$ and $c_{1}$, depending only on $a_{0}, w_{0}, d$ and $M$, such that if $\varepsilon \leqslant \varepsilon_{0}$ :

1. Equation (4) admits a particular solution $\bar{x}(\tau)$ with

$$
\left\|\bar{x}(\tau)-x^{\star}(\tau)\right\| \leqslant c_{1} \varepsilon \quad \forall \tau \in I .
$$

2. If $k \geqslant 3$, there exist functions $x_{j}(\tau), j=1, \ldots, k-2$ and a constant $c_{k-1}>0$ such that

$$
\left\|\bar{x}(\tau)-\left[x^{\star}(\tau)+\sum_{j=1}^{k-2} \varepsilon^{j} x_{j}(\tau)\right]\right\| \leqslant c_{k-1} \varepsilon^{k-1} \quad \forall \tau \in I .
$$


3. If $f$ is analytic in a complex neighbourhood of $x^{\star}(\tau)$, there exist functions $x_{j}(\tau), j \geqslant 1$, constants $c, K>0$ and an integer $N(\varepsilon)=\mathcal{O}(1 / \varepsilon)$ such that

$$
\left\|\bar{x}(\tau)-\left[x^{\star}(\tau)+\sum_{j=1}^{N(\varepsilon)} \varepsilon^{j} x_{j}(\tau)\right]\right\| \leqslant c \mathrm{e}^{-1 / K \varepsilon} \quad \forall \tau \in I .
$$

4. If $f$ is periodic in $\tau$ with period $T$, then $\bar{x}$ is also periodic with period $T$.

5. If all eigenvalues of $A(\tau)$ have a negative real part, there exist constants $M_{0}, c_{0}, \kappa>0$ such that any solution of (14) with initial condition such that $\left\|x\left(\tau_{0}\right)-x^{\star}\left(\tau_{0}\right)\right\| \leqslant c_{0}$ at some $\tau_{0} \in I$ satisfies

$$
\|x(\tau)-\bar{x}(\tau)\| \leqslant M_{0} \mathrm{e}^{-\kappa\left(\tau-\tau_{0}\right) / \varepsilon}\left\|x\left(\tau_{0}\right)-\bar{x}\left(\tau_{0}\right)\right\| \quad \forall \tau \in I \cap\left[\tau_{0}, \infty\right) .
$$

6. If A has eigenvalues with both positive and negative real part, there exist local invariant manifolds on which the motion is either contracting or expanding.

This result tells us that there exists indeed a particular solution tracking the equilibrium $x^{\star}(\tau)$ at a distance of order $\varepsilon$. This particular solution is sometimes called adiabatic or slow solution. It admits asymptotic series in $\varepsilon$ which can be computed by substitution into (国). If the equilibrium is asymptotically stable, the adiabatic solution attracts nearby solutions exponentially fast. In this case, the relation

$$
\lim _{\varepsilon \rightarrow 0} x(\tau ; \varepsilon)=x^{\star}(\tau) \quad \forall \tau>\tau_{0}
$$

implies that we may indeed take the formal limit $\varepsilon \rightarrow 0$ directly in (雨).

Theorem 1 has a long history. In the asymptotically stable case, points 1 . and 5 . were originally proved in [12, 13]. A different approach has been used in [14]. The exponential bounds in the analytic case are a result of an iterative scheme in [8], an alternative approach can be found in [15]. The periodicity of solutions is a consequence of the implicit function theorem, and the computation of invariant manifolds in the hyperbolic case is explained in [7]. This result can be extended to periodic orbits [16].

Let us also point out that this result has an interesting consequence for the linear equation

$$
\varepsilon \frac{\mathrm{d} y}{\mathrm{~d} \tau}=A(\tau) y,
$$

which is similar to the Schrödinger equation as appearing in the adiabatic theorem in quantum mechanics [17, 18]. This equation also occurs if we linearize (4) around any particular solution. The eigenvalues of the matrix $A(\tau) \in \mathbb{R}^{n \times n}$ can be labeled by continuous functions $a_{1}(\tau), \ldots, a_{n}(\tau)$.

Assumption 2. There exists a partition into two groups $\left\{a_{1}, \ldots, a_{p}\right\}$ and $\left\{a_{p+1}, \ldots, a_{n}\right\}$ such that the real gap

$$
\gamma=\inf _{\substack{\tau \in I \\ 1 \leqslant i \leqslant j \\ p+1 \leqslant j \leqslant n}}\left|\operatorname{Re}\left(a_{i}(\tau)-a_{j}(\tau)\right)\right|
$$

is strictly positive. 
Theorem $2(\llbracket 7)$. Assume that $A(\tau)$ is of class $\mathcal{C}^{3}$ for $\tau \in I$, and that Assumption 0 holds. For sufficiently small $\varepsilon$ and $\tau \in I$, there exists an invertible matrix $S(\tau, \varepsilon)$ such that the change of variables $y=S(\tau, \varepsilon) z$ transforms (10) into

$$
\varepsilon \dot{z}=D(\tau, \varepsilon) z,
$$

where $D(\tau, \varepsilon)$ is block-diagonal, with one block of size $p \times p$ and eigenvalues $a_{j}(\tau)+\mathcal{O}(\varepsilon)$ for $j=1, \ldots, p$, and another block of size $(n-p) \times(n-p)$ and eigenvalues $a_{j}(\tau)+\mathcal{O}(\varepsilon)$ for $j=p+1, \ldots, n$. The matrices $S(\tau, \varepsilon)$ and $D(\tau, \varepsilon)$ can be expanded into powers of $\varepsilon$, up to exponential order in the analytic case.

Since the connection between this result and Theorem 11 does not seem to be well known, we sketch the proof in Appendix A.

Corollary 1. Assume that the eigenvalues of $A(\tau, \varepsilon)$ have uniformly disjoint real parts, that is,

$$
\inf _{\substack{\tau \in I \\ 1 \leqslant j \leqslant n}}\left|\operatorname{Re}\left(a_{i}(\tau)-a_{j}(\tau)\right)\right|>0
$$

Then equation (10) can be diagonalized by a change of variables $y=S(\tau, \varepsilon) z$, and thus its principal solution can be written in the form

$$
U\left(\tau, \tau_{0}\right)=S(\tau, \varepsilon)\left(\begin{array}{ccc}
\mathrm{e}^{\alpha_{1}\left(\tau, \tau_{0}\right) / \varepsilon} & & 0 \\
& \ddots & \\
0 & & \mathrm{e}^{\alpha_{n}\left(\tau, \tau_{0}\right) / \varepsilon}
\end{array}\right) S\left(\tau_{0}, \varepsilon\right)^{-1},
$$

where

$$
\alpha_{j}\left(\tau, \tau_{0}\right)=\int_{\tau_{0}}^{\tau} a_{j}(s) \mathrm{d} s+\mathcal{O}(\varepsilon)
$$

\section{Single zero eigenvalue: hysteresis and scaling laws}

Theorem 11 breaks down if some eigenvalues of the linearization $A(\tau)=\partial_{x} f\left(x^{\star}(\tau), \tau\right)$ cross the imaginary axis, that is, in the case of a bifurcation. In this case, new phenomena may occur. As noted in [1], the center manifold theorem can be applied to the equation $\frac{\mathrm{d}}{\mathrm{d} t}(x, \tau, \varepsilon)=(f(x, \tau), \varepsilon, 0)$ to reduce the dimension of the system to the number of bifurcating eigenvalues.

The simplest case occurs when a single eigenvalue vanishes at the bifurcation point. We write the reduced equation in the form

$$
\varepsilon \frac{\mathrm{d} x}{\mathrm{~d} \tau}=f(x, \tau), \quad x \in \mathbb{R} .
$$

We shall assume that a stable equilibrium branch $x^{\star}(\tau)$, existing for negative $\tau$, bifurcates at the origin. This requires that $f(0,0)=\partial_{x} f(0,0)=0$.

Let us consider a few examples:

1. In the saddle-node bifurcation, the stable branch meets an unstable branch, and no equilibrium exists for positive $\tau$. This results in the adiabatic solution jumping to some other attractor. Jump phenomena were first studied in [19], and later in [20, 5, 1]. 
a

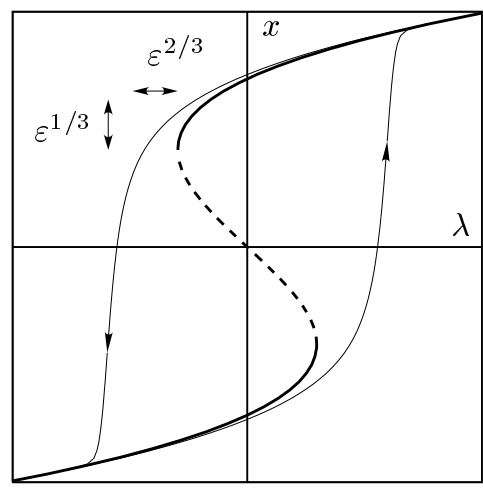

$\mathrm{b}$

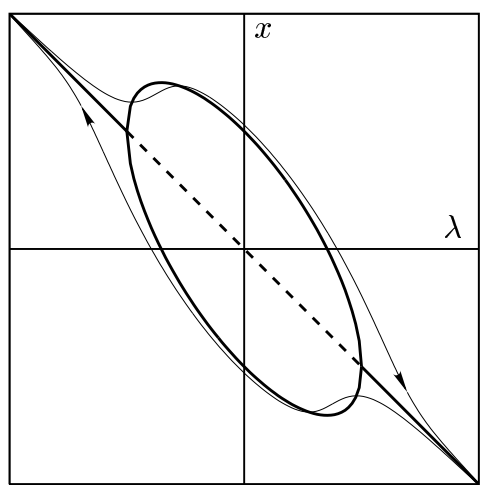

Figure 1. Comparison between solutions of equations with slowly moving parameters (light curves) and the bifurcation diagram of the associated frozen system (heavy curves, where full curves represent stable equilibria, and broken curves unstable ones). (a) The bifurcation diagram of equation (17) contains two saddle-node bifurcations. Solutions converge to a hysteresis cycle with area satisfying $\mathcal{A}(\varepsilon)-\mathcal{A}(0) \approx \varepsilon^{2 / 3}$. (b) The bifurcation diagram of equation (18) contains two pitchfork bifurcations. Solutions track the upper branch for increasing $\lambda$ and the lower one for decreasing $\lambda$, creating a hysteresis cycle with area satisfying $\mathcal{A}(\varepsilon)-\mathcal{A}(0) \approx \varepsilon^{3 / 4}$.

2. In the transcritical bifurcation, a stable and an unstable branch exchange stability. It has been shown in [21] that generically, solutions will track the outgoing stable branch, although it occasionally happens that the unstable branch is tracked for some time 22 .

3. In the pitchfork bifurcation, the stable branch becomes unstable, and two new stable branches are created. It has been shown in [23] that generically, solutions will track one of the two outgoing stable branches, where the choice of the branch depends on the geometry. If the original equilibrium does not depend on $\tau$, however, the bifurcation may be delayed [6, 22, 24].

These local properties of bifurcations have interesting global consequences if the parameter is varied periodically. The bifurcation points may be arranged in such a way that different branches are tracked for increasing and decreasing parameter, and thus there is hysteresis (and relation (9) no longer holds). A classic example is the equation

$$
\varepsilon \frac{\mathrm{d} x}{\mathrm{~d} \tau}=x-x^{3}+\lambda, \quad \lambda=\sin \tau,
$$

which leads to a familiar hysteresis curve (Fig. 1 1a). The curves $x(\tau)$ display so-called relaxation oscillations in which slow and fast motions alternate. Another example is

$$
\varepsilon \frac{\mathrm{d} x}{\mathrm{~d} \tau}=(x+\lambda)\left(1-\lambda^{2}-(x+\lambda)^{2}\right), \quad \lambda=2 \sin \tau,
$$

see Fig. 国b. In combination with other global mechanisms, such as inertia in the equation

$$
\varepsilon^{2} \frac{\mathrm{d}^{2} x}{\mathrm{~d} \tau^{2}}=-\gamma \varepsilon \frac{\mathrm{d} x}{\mathrm{~d} \tau}+\lambda(\tau) x-x^{3}, \quad \lambda=\sin \tau,
$$

hysteresis may also lead to a chaotic motion [6, 25].

Another interesting effect of bifurcations is that equilibrium branches are no longer tracked at a distance of order $\varepsilon$, but at a distance scaling with some fractional power of $\varepsilon$. This results in hysteresis areas scaling in nontrivial ways with $\varepsilon$ [26, 27, 28]. Similar 
scaling laws have been observed in ferromagnets [29, 30, and references therein], and they continue to be a domain of active research.

We present here a method to determine scaling laws in an easy, geometrical way [6, 7]. The key idea is to understand the behaviour of the variable $y=x-x^{\star}(\tau)$, which satisfies an equation of the form

$$
\varepsilon \frac{\mathrm{d} y}{\mathrm{~d} \tau}=a(\tau) y+b(y, \tau)+\varepsilon w(\tau)
$$

where $a(\tau)=\partial_{x} f\left(x^{\star}(\tau), \tau\right), w(\tau)=-\mathrm{d}_{\tau} x^{\star}(\tau)$ and $b(y, \tau)=\mathcal{O}\left(y^{2}\right)$.

Notation 1 . We write $x(\tau, \varepsilon) \approx y(\tau, \varepsilon)$ if there exist constants $c_{ \pm}>0$, uniform in $\tau$ and $\varepsilon$, such that $c_{-} y(\tau, \varepsilon) \leqslant x(\tau, \varepsilon) \leqslant c_{+} y(\tau, \varepsilon)$ for small $\tau$ and $\varepsilon$.

\section{Assumption 3.}

- The function $f(x, \tau)$ is of class $\mathcal{C}^{k}, k \geqslant 3$, for $0 \leqslant x \leqslant d$ and $\tau_{0} \leqslant \tau<0$, and admits the Taylor expansion

$$
f(x, \tau)=\sum_{n+m<k} c_{n m} x^{n} \tau^{m}+\sum_{n+m=k} R_{n m}(x, \tau) x^{n} \tau^{m}, \quad c_{00}=c_{10}=0 .
$$

- For $\tau_{0} \leqslant \tau<0$, there exists a function $x^{\star}(\tau) \approx|\tau|^{q}(q>0)$ such that $f\left(x^{\star}(\tau), \tau\right)=0$ and $f(x, \tau)<0$ for $x^{\star}(\tau)<x \leqslant d$.

- The function $a(\tau)=\partial_{x} f\left(x^{\star}(\tau), \tau\right)$ satisfies $a(\tau) \approx-|\tau|^{p}$, where

$$
p=\min _{n \geqslant 1, m \geqslant 0}\left\{q(n-1)+m \mid\left(n+m<k \text { and } c_{n m} \neq 0\right) \text { or } n+m=k\right\} .
$$

The numbers $q$ and $p$ can be determined geometrically by Newton's polygon, which is defined as the convex envelope of all points in $(n, m)$-plane such that either $n+m<k$ and $c_{n m} \neq 0$, or $n+m=k$. Then $-q$ is the slope of a segment of the polygon, and $p$ is the ordinate at 1 of this segment. The condition (22) is generically satisfied, as can be seen by inserting $x^{\star}(\tau) \approx|\tau|^{q}$ into the Taylor expansion (21).

Theorem 3 (《7). Under Assumption 月, any solution starting sufficiently close to $x^{\star}\left(\tau_{0}\right)$ reaches the $\mathcal{O}(\varepsilon)$-neighbourhood of $x^{\star}(\tau)$ at a time $\tau_{1}=\tau_{0}+\mathcal{O}(\varepsilon|\ln \varepsilon|)$ and satisfies

$$
x(\tau)-x^{\star}(\tau) \approx \begin{cases}\frac{\varepsilon}{|\tau|^{p+1-q}} & \text { for } \tau_{1} \leqslant \tau \leqslant-\varepsilon^{1 /(p+1)} \\ \varepsilon^{q /(p+1)} & \text { for }-\varepsilon^{1 /(p+1)} \leqslant \tau \leqslant 0 .\end{cases}
$$

The area between $x(\tau)$ and $x^{\star}(\tau)$ scales as

$$
\mathcal{A}(\varepsilon) \approx \begin{cases}\varepsilon^{(q+1) /(p+1)} & \text { if } q<p, \\ \varepsilon|\ln \varepsilon| & \text { if } q=p, \\ \varepsilon & \text { if } q>p .\end{cases}
$$

We sketch the proof in Appendix B. It is more difficult to study the behaviour of solutions after the bifurcation, where there is no such general result. One can, however, obtain much information by studying the dynamics of the distance between $x$ and the various equilibrium branches emerging from the bifurcation point, using similar methods.

For the branch of the pitchfork bifurcation with vertical tangent, we have $q=\frac{1}{2}$ and $p=1$, which implies that this branch is tracked at a distance of order $\varepsilon^{1 / 4}$, and the area scales as $\varepsilon^{3 / 4}$. For the saddle-node bifurcation, we have $q=p=\frac{1}{2}$. The branch is tracked at a distance of order $\varepsilon^{1 / 3}$, and the jump turns out to be delayed by a time scaling as $\varepsilon^{1 /(p+1)}=\varepsilon^{2 / 3}$, which accounts for the scaling law in Fig. 11a. 


\section{Hopf bifurcation and bifurcation delay}

We consider now the equation (田) in the case where a pair of complex conjugate eigenvalues crosses the imaginary axis from left to right. The frozen system generically displays one of the following two behaviours:

- in the supercritical Hopf bifurcation, a stable periodic orbit is created,

- in the subcritical Hopf bifurcation, an unstable periodic orbit is destroyed.

When the parameter is slowly varied through the bifurcation point, one would expect the appearance of oscillations with a slowly increasing amplitude in the first case, and a jump transition in the second case. This, however, does not occur if the system is smooth enough: the moment at which the trajectory departs from the unstable equilibrium turns out to be delayed with respect to the moment of the bifurcation (Fig. 2a). This bifurcation delay was first described in [31], analysed rigorously by Neishtadt [8, 9], and observed in various physical systems 32, 33. We now state a version of Neishtadt's result on the computation of the delay.

Assumption 4. There exist an open interval $I \in \mathbb{R}$ containing 0 and a neighbourhood $\mathcal{D}$ of the origin in $\mathbb{R}^{n}$ such that

- The function $f(x, \tau)$ is analytic in a complex neighbourhood of $I \times \mathcal{D}$.

- There is a curve $x^{\star}(\tau): I \rightarrow \mathbb{R}^{n}$ with $f\left(x^{\star}(\tau), \tau\right)=0$.

- The matrix $A(\tau)=\partial_{x} f\left(x^{\star}(\tau), \tau\right)$ has two eigenvalues $a(\tau) \pm \mathrm{i} \omega(\tau)$, where $a(0)=0$, $a^{\prime}(0)>0, \omega(0) \neq 0$, and $a(\tau)$ has the same sign as $\tau$; all other eigenvalues of $A(\tau)$ have a strictly negative real part.

For $\tau$ in a complex neighbourhood of $I$, we can define the function

$$
\Psi(\tau)=\int_{0}^{\tau}[a(s)+\mathrm{i} \omega(s)] \mathrm{d} s .
$$

For negative $\tau_{0} \in I$, we define the function

$$
\Pi\left(\tau_{0}\right)=\sup _{\tau>\tau_{0}}\left\{\tau \mid \operatorname{Re} \Psi(s)<\operatorname{Re} \psi\left(\tau_{0}\right), \tau_{0}<s<\tau\right\} .
$$

We have $\Pi\left(\tau_{0}\right)>0$ for $\tau_{0}<0$ because $a(s)$ is negative for negative $\tau$, which implies that $\operatorname{Re} \Psi(\tau)$ is decreasing for negative $\tau$. Moreover, we have $\lim _{\tau_{0} \rightarrow 0-} \Pi\left(\tau_{0}\right)=0$ and $\lim _{\tau_{0} \rightarrow 0-} \Pi^{\prime}\left(\tau_{0}\right)=-1$. For instance, if $a(\tau)=\tau$, then $\Pi\left(\tau_{0}\right)=-\tau_{0}$.

Theorem 4 (《8). For any strictly negative $\tau_{0} \in I$ there exist constants $\varepsilon_{0}, c_{0}, c_{1}>0$ and a continuous function $r(\varepsilon)$ with $\lim _{\varepsilon \rightarrow 0} r(\varepsilon)=0$ such that for $\varepsilon<\varepsilon_{0}$, any solution with initial condition such that $\left\|x\left(\tau_{0}\right)-x^{\star}\left(\tau_{0}\right)\right\| \leqslant c_{0}$ satisfies

$$
\left\|x(\tau)-x^{\star}(\tau)\right\| \leqslant c_{1} \varepsilon \quad \text { for } \tau_{0}+r(\varepsilon) \leqslant \tau \leqslant \min \left\{\tau_{+}, \Pi\left(\tau_{0}\right)\right\}-r(\varepsilon) .
$$

The so-called buffer time $\tau_{+}>0$ depends only on the behaviour of the eigenvalue $a(\tau)+$ $\mathrm{i} \omega(\tau)$ in the complex plane.

The computation of the buffer time $\tau_{+}$is explained in [9], see also [3]. Roughly speaking, it is the largest real time which can be connected to the negative real axis by a path with constant $\operatorname{Re} \Psi$. It is usually of the order of $\omega(0)$. The remarkable fact is that unlike for the pitchfork bifurcation, the bifurcation delay exists also when the equilibrium $x^{\star}(\tau)$ depends on $\tau$. 

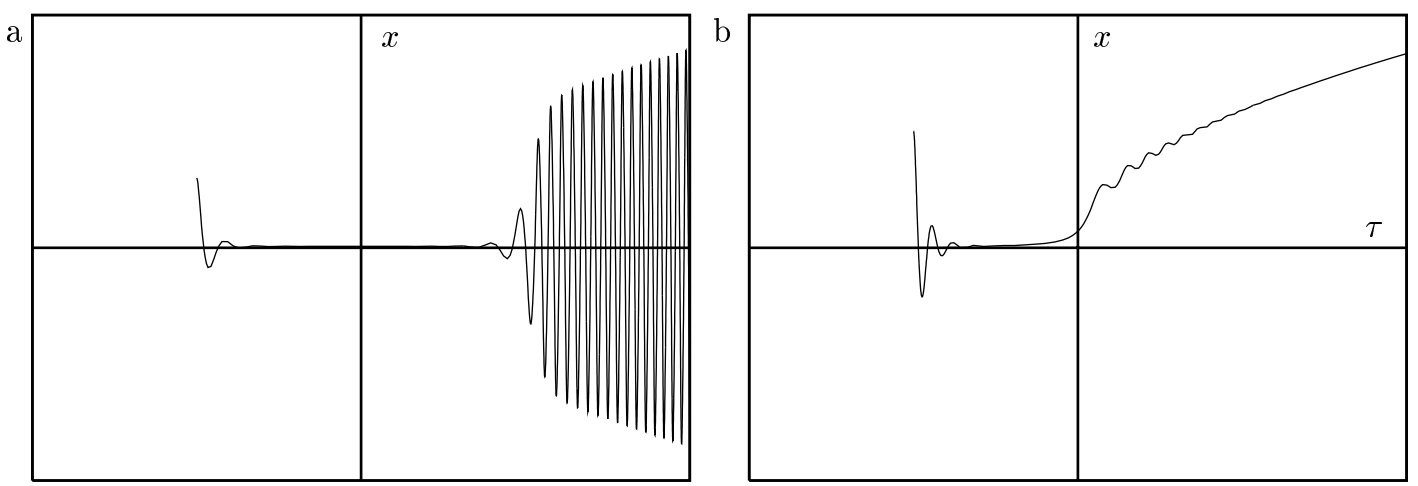

FIgURE 2. (a) The slow passage through a Hopf bifurcation (occurring at the origin) leads to the delayed appearance of oscillations. The delay depends on the initial condition, but admits a saturation value, called buffer time. (b) The delay is suppressed by an appropriate feedback control, which creates a bifurcation with double zero eigenvalue. Solutions track a stable equilibrium branch emerging from the bifurcation point.

\section{Double zero eigenvalue and feedback control}

Jumps and large amplitude oscillations may have catastrophic consequences for a device undergoing bifurcations. This has lead some researchers to design feedback controlled systems. A simple affine control system takes the form

$$
\frac{\mathrm{d} x}{\mathrm{~d} t}=f(x, \lambda)+b u(x, \lambda),
$$

where $f(x, \lambda)$ is the uncontrolled vector field satisfying Assumption $\llbracket, b \in \mathbb{R}^{n}$ is a given vector, which defines the direction in which the system can be steered, and $u(x, \lambda) \in \mathbb{R}$ is the scalar feedback control. In previous works [34, 35, 36], feedback controls have been designed in order to make the bifurcation supercritical, so that orbits departing from the unstable equilibrium have a bounded amplitude.

The results of the previous section show however that in the case of a dynamic Hopf bifurcation, a jump transition occurs even in the supercritical case. To avoid such a jump, one may try to suppress the bifurcation delay by moving the imaginary parts of the bifurcating eigenvalues to zero, in order to push the buffer time to zero. This will produce a bifurcation with double zero eigenvalue. Moreover, the control should ensure that the system is nonlinearly stable at the bifurcation point, in such a way that stable equilibria are created which attract the solutions.

In [10] we construct a feedback control which satisfies these requirements. We then show that the dynamics can be reduced to the two-dimensional equation

$$
\begin{aligned}
& \varepsilon \frac{\mathrm{d} x}{\mathrm{~d} \tau}=y \\
& \varepsilon \frac{\mathrm{d} y}{\mathrm{~d} \tau}=\mu(\tau) x+2 a(\tau) y+\gamma(\tau) x^{2}+\delta(\tau) x y-x^{2} y-x^{3}+\mathcal{O}\left(\left(x^{2}+y^{2}\right)^{2}\right)+\varepsilon R(x, y, \tau, \varepsilon),
\end{aligned}
$$

where $a(\tau)$ is the same as in Assumption 4, the functions $\mu(\tau), \gamma(\tau)$ and $\delta(\tau)$ vanish at $\tau=0$ and $R(0,0, \tau, 0)$ is directly related to the drift of $x^{\star}(\tau)$. The linearization of (29) at the origin has eigenvalues $a(\tau) \pm \mu(\tau)^{1 / 2}$. The frozen version of this system is a codimension-four unfolding of the singular vector field $\left(y,-x^{2} y-x^{3}\right)$ which has been studied in detail [37]. 
Theorem 5 (10]). Assume that the right-hand-side of (29) is analytic, $a^{\prime}(0)>0$ and $\mu^{\prime}(0)>0$. There exist positive constants $d, T, M, \kappa$ and a neighbourhood $\mathcal{N}$ of the origin in $\mathbb{R}^{2}$ with the following property. For every $\tau_{0} \in[-T, 0)$, there is a constant $c_{1}>0$ such that for sufficiently small $\varepsilon$, any solution of (29) with initial condition $(x, y)\left(\tau_{0}\right) \in \mathcal{N}$ satisfies

$$
\begin{array}{lll}
|x(\tau)| \leqslant M \varepsilon|\tau|^{-1}, & |y(\tau)| \leqslant M \varepsilon|\tau|^{-1 / 2}, & \tau_{1}(\varepsilon) \leqslant \tau \leqslant-(\varepsilon / d)^{2 / 3}, \\
|x(\tau)| \leqslant M \varepsilon^{1 / 3}, & |y(\tau)| \leqslant M \varepsilon^{2 / 3}, & -(\varepsilon / d)^{2 / 3} \leqslant \tau \leqslant(\varepsilon / d)^{2 / 3},
\end{array}
$$

where $\tau_{1}(\varepsilon)=\tau_{0}+c_{1} \varepsilon|\ln \varepsilon|$. If, moreover, the relations $\mu^{\prime}(0)>2 a^{\prime}(0)$ and $R(0,0,0,0) \neq 0$ hold, then for $(\varepsilon / d)^{2 / 3} \leqslant \tau \leqslant T$ we have

$$
\begin{array}{r}
\left|x(\tau)-x_{+}(\tau)\right| \leqslant M\left[\varepsilon \tau^{-1}+\varepsilon^{1 / 2} \tau^{-1 / 4} \mathrm{e}^{-\kappa \tau^{2} / \varepsilon}\right], \\
|y(\tau)| \leqslant M\left[\varepsilon \tau^{-1 / 2}+\varepsilon^{1 / 2} \tau^{1 / 4} \mathrm{e}^{-\kappa \tau^{2} / \varepsilon}\right],
\end{array}
$$

where

$$
x_{+}(\tau)= \begin{cases}\sqrt{\mu}+\mathcal{O}(\tau), & \text { if } R(0,0,0,0)>0, \\ -\sqrt{\mu}+\mathcal{O}(\tau), & \text { if } R(0,0,0,0)<0\end{cases}
$$

are equilibria of (29), i.e., the right-hand side of (29) vanishes when $x=x_{+}, y=0$ and $\varepsilon=0$.

This behaviour is illustrated in Fig. 2 $\mathrm{b}$. The important fact is that instead of tracking the unstable equilibrium (represented by the origin) for some time, the trajectory smoothly tracks the equilibrium $x_{+}(\tau)$, a feature that can be used to detect the fact that a bifurcation has occurred. The behaviour is more complicated if $\mu^{\prime}(0)<2 a^{\prime}(0)$ 10, 11.

\section{Acknowledgments}

The research presented in Sections 2 and 3 was done in collaboration with Hervé Kunz, and partly supported by the Swiss National Fund. Results in Section 5 were obtained in collaboration with Klaus Schneider, and supported by the Nonlinear Control Network of the European Community, Grant ERB FMRXCT-970137.

\section{A Proof of Theorem 2}

If $A(\tau)$ is of class $\mathcal{C}^{k}$ and satisfies Assumption 2, there exists a matrix $S_{0}(\tau)$ of class $\mathcal{C}^{k}$ such that $S_{0}^{-1} A S_{0}=D_{0}$ is block-diagonal [38, 39]. The change of variables $y=S_{0}(\tau) y_{0}$ yields the equation

$$
\varepsilon \frac{\mathrm{d} y_{0}}{\mathrm{~d} \tau}=A_{0}(\tau) y_{0}, \quad A_{0}(\tau ; \varepsilon)=S_{0}^{-1} A S_{0}-\varepsilon S_{0}^{-1} \frac{\mathrm{d} S_{0}}{\mathrm{~d} \tau}=\left(\begin{array}{cc}
A_{11} & \varepsilon A_{12} \\
\varepsilon A_{21} & A_{22}
\end{array}\right) .
$$

This equation can be transformed into the block-diagonal form (12) by the change of variables $y_{0}=S_{1}(\tau) z$ if we impose that $S_{1}$ satisfy the differential equation

$$
\varepsilon \frac{\mathrm{d} S_{1}}{\mathrm{~d} \tau}=A_{0}(\tau) S_{1}-S_{1} D(\tau)
$$


We will look for a solution with matrices of the form

$$
S_{1}(\tau)=\left(\begin{array}{cc}
\mathbb{1}_{p} & \varepsilon S_{12} \\
\varepsilon S_{21} & \mathbb{1}_{n-p}
\end{array}\right), \quad D(\tau)=\left(\begin{array}{cc}
D_{1} & 0 \\
0 & D_{2}
\end{array}\right)
$$

where $S_{12} \in \mathbb{R}^{p \times(n-p)}$ and $S_{21} \in \mathbb{R}^{(n-p) \times p}$. Substitution into (35) leads to

$$
\varepsilon \frac{\mathrm{d} S_{12}}{\mathrm{~d} \tau}=A_{12}+A_{11} S_{12}-S_{12} A_{22}-\varepsilon^{2} S_{12} A_{21} S_{12}, \quad D_{2}=A_{22}+\varepsilon^{2} A_{21} S_{12}
$$

and similar relations for $S_{21}, D_{1}$. Let $L$ be the linear operator $L: X \mapsto A_{11} X-X A_{22}$. For $\varepsilon=0$, the eigenvalues of $L$ are exactly given by $a_{i}-a_{j}, 1 \leqslant i \leqslant p, p+1 \leqslant j \leqslant n$ [38, 39]. Assumption 2 implies that $L$ is invertible (its inverse can be represented as a complex integral [40]), and by the implicit function theorem, the right-hand-side of (37) vanishes for $S_{12}=S^{\star}(\tau)=-L^{-1} A_{12}+\mathcal{O}(\varepsilon)$. The Fréchet derivative of (37) around $S^{\star}$ is equal to $L+\mathcal{O}\left(\varepsilon^{2}\right)$, and thus, by Assumption 2 , $S^{\star}$ is hyperbolic for small $\varepsilon$. Theorem 1 can then be applied to show the existence of a solution $S_{12}(\tau)=S^{\star}(\tau)+\mathcal{O}(\varepsilon)$.

\section{B Proof of Theorem 3}

The assertion on $\tau_{1}$ is a consequence of Theorem 11. Let $\mu=\frac{q}{p+1}$ and $\nu=\frac{1}{p+1}$. We first consider equation (20) for $\tau_{1} \leqslant \tau \leqslant-\varepsilon^{\nu}$. By assumption, we have $a(\tau) \leqslant-a_{+}|\tau|^{p}$ for some $a_{+}>0$. Let $b_{0}>0$. Since $y\left(\tau_{1}\right)=\mathcal{O}(\varepsilon)$, there exists by continuity a time $\tau^{\star} \in\left(\tau_{1}, 0\right]$ such that $y(\tau) \leqslant a_{+}|\tau|^{q} / 2 b_{0}$ for $\tau_{1} \leqslant \tau \leqslant \tau^{\star}$. Moreover, we may assume that either $\tau^{\star}=-\varepsilon^{\nu}$ or $y\left(\tau^{\star}\right)=a_{+}|\tau|^{q} / 2 b_{0}$. Using the Tayor expansion of $f$, one can show that for $\tau \in\left[\tau_{1}, \tau^{\star}\right], b(y, \tau) \leqslant b_{0} y^{2}|\tau|^{p-q}$, provided $b_{0}$ is large enough. Then for $\tau_{1} \leqslant \tau \leqslant \tau^{\star}$

$$
\varepsilon \frac{\mathrm{d} y}{\mathrm{~d} \tau} \leqslant-\frac{a_{+}}{2 b_{0}}|\tau|^{p} y+\varepsilon w_{0}|\tau|^{q-1} .
$$

This linear equation can be solved explicitly, to prove that $y(\tau) \leqslant c_{+} \varepsilon||^{q-p-1}$ on the time interval under consideration, for some $c_{+}>0$. Let us write $\tau^{\star}=-\varepsilon^{\nu-\delta}, \delta \geqslant 0$. Then $y\left(\tau^{\star}\right) \leqslant c_{+} \varepsilon^{\delta(p+1)}\left|\tau^{\star}\right|^{q}$. By definition of $\tau^{\star}$, we have either $\tau^{\star}=-\varepsilon^{\nu}$, or $y\left(\tau^{\star}\right)=$ $a_{+}\left|\tau^{\star}\right|^{q} / 2 b_{0}$, and then $\varepsilon^{\delta(p+1)} \geqslant a_{+} / 2 b_{0} c_{+}$, which shows that $\tau^{\star} \geqslant-\left(2 b_{0} c_{+} / a_{+}\right)^{\nu} \varepsilon^{\nu} \approx-\varepsilon^{\nu}$. One obtains a lower bound in the same way, which proves that $y(\tau) \approx \varepsilon|\tau|^{q-p-1}$ for $\tau_{1} \leqslant \tau \leqslant \tau^{\star} \approx-\varepsilon^{\nu}$

For $\tau^{\star} \leqslant \tau \leqslant 0$, the rescaling $y=\varepsilon^{\mu} z, \tau=\varepsilon^{\nu} \sigma$ gives

$$
\frac{\mathrm{d} z}{\mathrm{~d} \sigma}=\tilde{a}(\sigma) z+\tilde{b}(z, \sigma)+\tilde{w}(\sigma)
$$

where $\tilde{a}(\sigma)=\varepsilon^{\nu-1} a\left(\varepsilon^{\nu} \sigma\right) \approx-|\sigma|^{p}, \tilde{w}(\sigma)=\varepsilon^{\nu-\mu} w\left(\varepsilon^{\nu} \sigma\right) \approx|\sigma|^{q-1}$, and one can show with the Taylor expansion that $\tilde{b}(\sigma, z)=\varepsilon^{\nu-\mu-1} b\left(\varepsilon^{\mu} z, \varepsilon^{\nu} \sigma\right)$ is smaller than a constant which does not depend on $\varepsilon$. Using the fact that $\mathrm{d}_{\sigma} z \geqslant-a_{-}|\sigma|^{p} z-b_{0} z^{2}$, we obtain by solving this Bernoulli equation that $z$ is bounded below by a positive constant uniform in $\varepsilon$. Moreover, the hypothesis $f\left(x^{\star}(\tau)+y, \tau\right)<0$ for $y>0$ shows that $\tilde{a}(\sigma) z+\tilde{b}(z, \sigma)<0$ for $z>0$, which yields the upper bound $\mathrm{d}_{\sigma} z \leqslant w_{0}|\sigma|^{q-1}$. This shows that $z(\sigma)$ is also bounded from above by a constant independent of $\varepsilon$. This proves that $y(\tau) \approx \varepsilon^{\mu}$ for $\tau^{\star} \leqslant \tau \leqslant 0$. 


\section{References}

[1] E.F. Mishchenko, Yu.S. Kolesov, A.Yu. Kolesov, N.Kh. Rozov, Asymptotic Methods in Singularly Perturbed Systems (Consultants Bureau, New York, 1994).

[2] A.B. Vasil'eva, V.F. Butusov, L.V. Kalachev, The Boundary Function Method for Singular Perturbation Problems (SIAM, Philadelphia, 1995).

[3] E. Benoît (Ed.), Dynamic Bifurcations, Proc. (Luminy, 1990) (Lecture Notes in Math. 1493, Springer, Berlin, 1991).

[4] C.K.R.T. Jones, Geometric Singular Perturbation Theory, in R. Johnson (Ed.), Dynamical Systems, Proc. (Montecatini Terme, 1994) (Lecture Notes in Math. 1609, Springer, Berlin, 1995).

[5] E.F. Mishchenko, N.Kh. Rozov, Differential Equations with Small Parameters and Relaxations Oscillations (Plenum, New York, 1980).

[6] N. Berglund, H. Kunz, Memory Effects and Scaling Laws in Slowly Driven Systems, J. Phys. A 32 (1999), 15-39.

[7] N. Berglund, Adiabatic Dynamical Systems and Hysteresis, Thesis EPFL no 1800 (1998). Available at http://dpwww.epfl.ch/instituts/ipt/berglund/these.html

[8] A.I. Neishtadt, Persistence of stability loss for dynamical bifurcations I, II, Diff. Equ. 23 (1987), 1385-1391. Diff. Equ. 24 (1988), 171-176.

[9] A.I. Neishtadt, On Calculation of Stability Loss Delay Time for Dynamical Bifurcations in D. Jacobnitzer Ed., XI ${ }^{\text {th }}$ International Congress of Mathematical Physics (International Press, Boston, 1995).

[10] N. Berglund, Control of dynamic Hopf bifurcations. To appear in Nonlinearity 13 (January 2000).

[11] N. Berglund, K.S. Schneider, Control of dynamic bifurcations, in D. Aeyels, F. LamnabhiLagarrigue, A. van der Schaft (Eds.), Stability and Stabilization of Nonlinear Systems (Lecture Notes in Contr. and Inform. Sciences 246, Springer, Berlin, 1999).

[12] A.N. Tihonov, Systems of differential equations containing small parameters in the derivatives, Mat. Sbornik N.S. 31 (1952), 575-586.

[13] I.S. Gradšteĭn, Applications of A.M. Lyapunov's theory of stability to the theory of differential equations with small parameters in the derivatives, Mat. Sbornik N.S. 32 (1953), 263-286.

[14] N. Fenichel, Geometric singular perturbation theory for ordinary differential equations, J. Diff. Eq. 31 (1979), 53-98.

[15] C. Baesens, Gevrey series and dynamic bifurcations for analytic slow-fast mappings, Nonlinearity 8 (1995), 179-201.

[16] L.S. Pontryagin, L.V. Rodygin, Approximate solution of a system of ordinary differential equations involving a small parameter in the derivatives, Dokl. Akad. Nauk SSSR 131 (1960), 237-240.

[17] M.V. Berry, Histories of Adiabatic Quantum Transitions, Proc. Roy. Soc. London A 429 (1990), 61-72.

[18] A. Joye, H. Kunz, C.-E. Pfister, Exponential decay and geometric aspects of transition probabilities in the adiabatic limit, Ann. Physics 208 (1991), 299-332.

[19] L.S. Pontryagin, Asymptotic behavior of solutions of systems of differential equations with a small parameter in the derivatives of highest order, Izv. Akad. Nauk SSSR. Ser. Mat. 21 (1957), 605-626.

[20] R. Haberman, Slowly varying jump and transition phenomena associated with algebraic bifurcation problems, SIAM J. Appl. Math. 37 (1979), 69-106. 
[21] N.R. Lebovitz, R.J. Schaar, Exchange of Stabilities in Autonomous Systems, Stud. in Appl. Math. 54 (1975), 229-260.

[22] N.N. Nefedov, K.R. Schneider, Delayed exchange of stabilities in singularly perturbed systems, Zeitschr. Angewandte Mathematik und Mechanik (ZAMM) 78 (1998), 199-202.

[23] N.R. Lebovitz, R.J. Schaar, Exchange of Stabilities in Autonomous Systems II, Stud. in Appl. Math. 56 (1977), 1-50.

[24] T. Erneux, P. Mandel, Imperfect bifurcation with a slowly-varying control parameter, SIAM J. Appl. Math. 46 (1986), 1-15.

[25] N. Berglund, H. Kunz, Chaotic Hysteresis in an Adiabatically Oscillating Double Well, Phys. Rev. Letters 78 (1997), 1691-1694.

[26] P. Jung, G. Gray, R. Roy, P. Mandel, Scaling Law for Dynamical Hysteresis, Phys. Rev. Letters 65 (1990), 1873-1876.

[27] A. Hohl, H.J.C. van der Linden, R. Roy, G. Goldsztein, F. Broner, S.H. Strogatz, Scaling Laws for Dynamical Hysteresis in a Multidimensional Laser System, Phys. Rev. Letters $\mathbf{7 4}$ (1995), 2220-2223.

[28] G.H. Goldsztein, F. Broner, S.H. Strogatz, Dynamical Hysteresis without Static Hysteresis: Scaling Laws and Asymptotic Expansions, SIAM J. Appl. Math. 57 (1997), 1163-1187.

[29] M. Rao, H.K. Krishnamurthy, R. Pandit, Magnetic hysteresis in two model spin systems, Phys. Rev. B 42 (1990), 856-884.

[30] J.-S. Suen, J.L. Erskine, Magnetic Hysteresis Dynamics: Thin $p(1 \times 1)$ Fe Films on Flat and Stepped W(110), Phys. Rev. Letters 78 (1997), 3567-3570.

[31] M.A. Shishkova, Examination of one system of differential equations with a small parameter in highest derivatives, Dokl. Akad. Nauk SSSR 209 (1973), 576-579. [English transl.: Soviet Math. Dokl. 14 (1973), 384-387].

[32] S.M. Baer, T. Erneux, J. Rinzel, The slow passage through a Hopf bifurcation: delay, memory effects, and resonance, SIAM J. Appl. Math. 49 (1989), 55-71.

[33] L. Holden, T. Erneux, Slow passage through a Hopf bifurcation: From oscillatory to steady state solutions, SIAM J. Appl. Math. 53 (1993), 1045-1058.

[34] D. Aeyels, Stabilization of a class of nonlinear systems by a smooth feedback control, Systems Control Lett. 5 (1985), 289-294.

[35] E.H. Abed, Bifurcation-theoretic issues in the control of voltage collapse, in J.H. Chow (Ed.), Systems and control theory for power systems (Springer, New York, 1995).

[36] W. Müller, K.R. Schneider, Feedback Stabilization of Nonlinear Discrete-Time Systems, J. Differ. Equations Appl. 4 (1998), 579-596.

[37] A.I. Khibnik, B. Krauskopf, C. Rousseau, Global study of a family of cubic Liénard equations, Nonlinearity 11 (1998), 1505-1519.

[38] W. Wasow, Asymptotic expansions for ordinary differential equations (Krieger, New York, 1965, 1976).

[39] R. Bellman, Introduction to Matrix Analysis (McGraw-Hill, New York, 1960).

[40] S.G. Krein, Linear Differential Equations in Banach Spaces (American Mathematical Society, Providence, Rhode Island, 1971). 